\title{
45-Year-Old Female with a 25 Year History of Seizures
}

\author{
Fahd M. AlSufiani, Jorge G. Burneo, Richard S. McLachlan, \\ David M. Pelz, David A. Steven, Robert R. Hammond
}

Can J Neurol Sci. 2013; 40: 85-88

\section{Case Presentation: Dr. Fahd AlSufiani}

A 45-year-old right hand dominant female presented for continued investigation and treatment of spells that occurred intermittently over the past 25 years.

Her stereotyped events begin with an ill-defined upper abdominal discomfort accompanied by nausea and a sensation of heat rising upwards from her abdomen. On occasion she experiences the illusion of watching herself from outside her body.

The spells usually last 30 to 60 seconds, without loss of awareness. On the rare occasion when she loses awareness, witnesses describe that she stops what she is doing and stares blankly. Slight chewing motions have been noted in association with some spells. Afterwards, she describes a sense of fatigue with variable mild confusion and occasional headache. She suffers approximately eight to ten episodes a month with a greater frequency near menses and at times of stress.

Her perinatal and developmental histories were normal. She had no abnormalities of speech or memory and no history of head trauma, central nervous system (CNS) infections or febrile seizures. She completed high school with average to above average grades.

There is no family history of neurological disease. She has three healthy children. She consumes alcohol on occasion and is a non-smoker. She works on a poultry farm.

On examination she is in no acute distress, afebrile and has stable vital signs (HR 78, RR 18, BP 124/70). She is $160 \mathrm{~cm}$ in height and weighs $91 \mathrm{~kg}$. She is alert and oriented. Head and neck, cranial nerve, motor, sensory and cerebellar examinations are all normal.

\section{Dr. Jorge Burneo}

This 45-year-old female is experiencing events that are characterized by sudden onset, short duration, and they are stereotypical, indicating that they are most likely the result of excessive or synchronous neuronal activity in the cortex. Even though the differential diagnosis of this type of event might include migraines, psychosis, and psychogenic non-epileptic events, the diagnosis of epileptic seizures is obvious.

Based on the new seizure terminology $y^{1}$ the patient was experiencing autonomic and dyscognitive seizures (simple and complex partial seizures).

Her abdominal aura, with the upward migratory pattern, and the associated experiential phenomenon (watching herself), loss of awareness with staring, oral automatism, and postictal confusion indicate a limbic involvement, most likely mesial temporal in onset. Other possible localizations may include the orbito-frontal region and the cingulate gyrus, which are less likely. Despite the clear localization, it is important to mention that the symptomatology experienced by the patient may represent 'spread' from a different area (epileptogenic focus). Areas that frequently spread to the mesial temporal region include the neocortical temporal area, occipital and orbitofrontal regions.

Unfortunately, I cannot lateralize the onset of the seizures. Hence, my initial impression would be localization-related epilepsy manifested by autonomic and dyscognitive seizures of possible temporal lobe origin.

The possible list of aetiologies include hippocampal sclerosis (the most common aetiology of temporal lobe epilepsy), lowgrade glioneuronal tumours (particularly ganglioglioma and DNET), malformations of cortical development or $\mathrm{MCDs}^{2}$ (cortical dysplasia, focal nodular heterotopia, polymicrogyria), vascular (cavernoma), and non-lesional (or 'cryptogenic').

At this point it would be necessary to obtain more information about trials of antiepileptic drugs (AEDs), and previous investigations done before she was referred to our center.

\section{Dr. AlSufiani}

The patient had been treated in the remote past with phenytoin but was switched to levetiracetam in recent years. Her current dosing was $500 \mathrm{mg}$ in the morning and $1000 \mathrm{mg}$ in the evening.

From the Department of Pathology, Division of Neuropathology (FMA, RRH), Epilepsy Program (JGB, RSM, DAS), Division of Neurosurgery (DAS), Department of Clinical

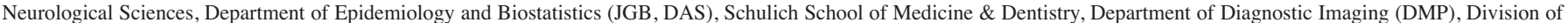
Neuroradiology, London Health Sciences Centre and the University of Western Ontario, London, Ontario, Canada.

Received January 12, 2012. Final Revisions Submitted July 20, 2012.

Reprint requests to: Robert Hammond, Rm. A-148, Department of Pathology, LHSC-UH, 339 Windermere Road, London, Ontario, N6A 5A5, Canada.

Email: RobertHammond@lhsc.on.ca 


\section{Dr. BURNeO}

Because the patient has already failed two AEDs, she would be considered medically-refractory. ${ }^{3}$ She would be a candidate for presurgical investigation in a comprehensive epilepsy program, in order to confirm the diagnosis and assess her candidacy for epilepsy surgery. The investigation will need to include scalp video electroencephalography (EEG), magnetic resonance imaging (MRI) of the brain, and neuropsychological evaluation. Depending on initial results, she might need other studies like single photon emission computed tomography (SPECT), positron emissions tomography (PET), and/or intracarotid amobarbital testing.

\section{Dr. MCLACHLAN}

Scalp EEG studies revealed right temporal-frontal spikes and nonspecific right temporal-frontal and generalized slowing (Figure 1). There was some exacerbation of these abnormalities with hyperventilation but not with photic stimulation. The origin of the abnormalities was felt to be right temporal, but a frontal origin could not be excluded.

The patient was admitted to the Epilepsy Monitoring Unit for further monitoring and scalp video-EEG recordings. During her stay, five clinical seizures were recorded that exhibited typical semiology. Their localization by EEG suggested a right temporal origin, however the possibility of a right frontal origin with temporal propagation could not be excluded.

\section{Dr. AlSufiani}

Neuropsychological testing revealed high-average intellect with intact visual and verbal memory and mild frontal lobe dysfunction. There were no depressive symptoms.

\section{Dr. Pelz}

Sequential MRI examinations had followed a right inferior temporal lesion that had not changed appreciably in size for more than 20 years since her first imaging studies. The partly cystic lesion measured approximately $2.5 \mathrm{~cm}$ in diameter and did not enhance (Figure 2).

\section{Dr. BURNEO}

The lesion in the MRI appears to be compatible with a lowgrade tumor, most likely a ganglioglioma. Due to the lack of definitive localization of the seizure focus, despite the presence of a lesion in the MRI, the patient required either an interictal and ictal SPECT study or further monitoring in the Epilepsy Unit with intracranially-placed subdural electrodes covering the temporal (mesial and neocortical) and frontal (orbito-frontal and convexity) regions of the right cerebral hemisphere.

\section{Dr. MCLachlan}

Invasive EEG recording was facilitated by Dr. Steven, using subdurally-placed electrodes covering the right temporal and frontal lobes. The latter studies recorded abundant spikes emanating from the right anterior mesial temporal region. A further six stereotypic seizures were captured, with definitive localization in the right anterior mesial temporal lobe with late right frontal propagation (Figure 3).

\section{Dr. BURNeO}

Based on the video-EEG recordings with intracraniallyplaced subdural electrodes, the patient requires a standard anterior right temporal lobectomy, including mesial structures (including amygdalohippocampectomy), and the removal of all abnormal tissue, if technically possible. In this scenario, surgical intervention would be favoured over continuation of medical

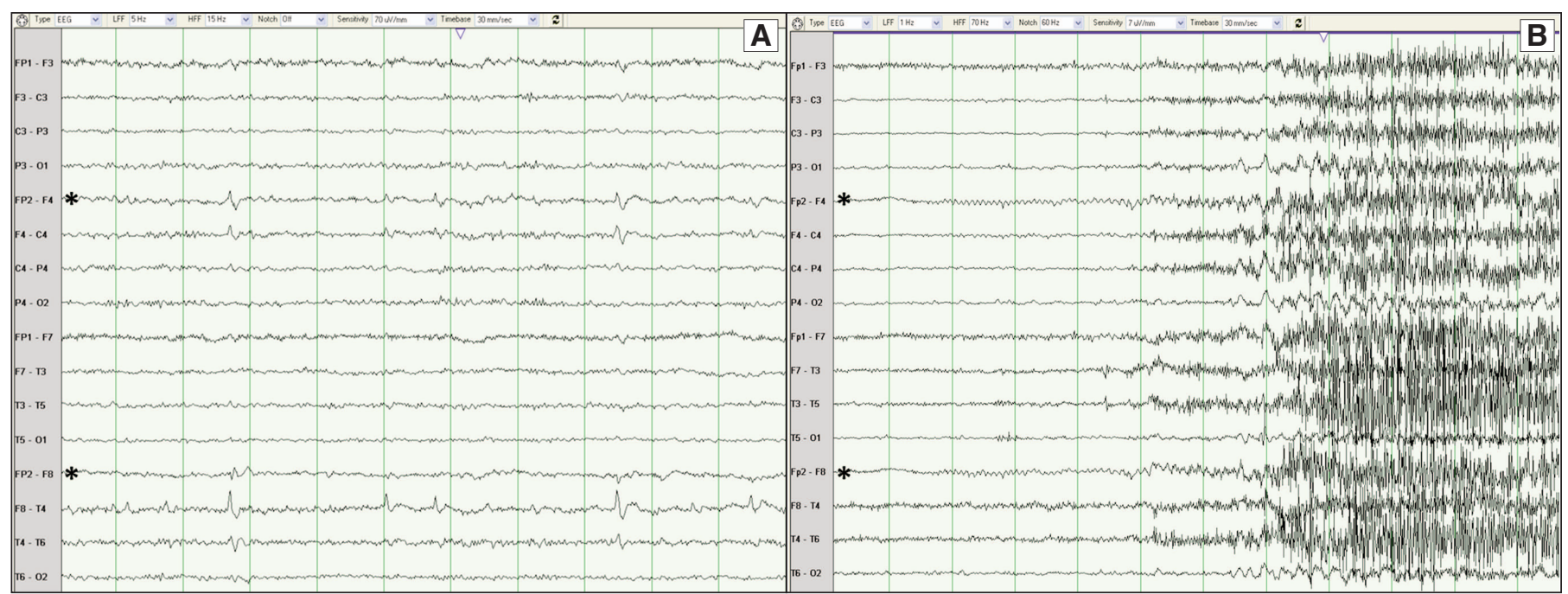

Figure 1: a) Interictal EEG during wakefulness shows frequent right temporal-frontal spikes and focal slowing in the same area. b) Seizure onsets (midpage) as right temporal frontal low voltage 8 hertz rhythmic waves (channels 5 and 13, asterisks) evolving over two seconds to right hemisphere 2.5 hertz waves and diffuse muscle artefact. 

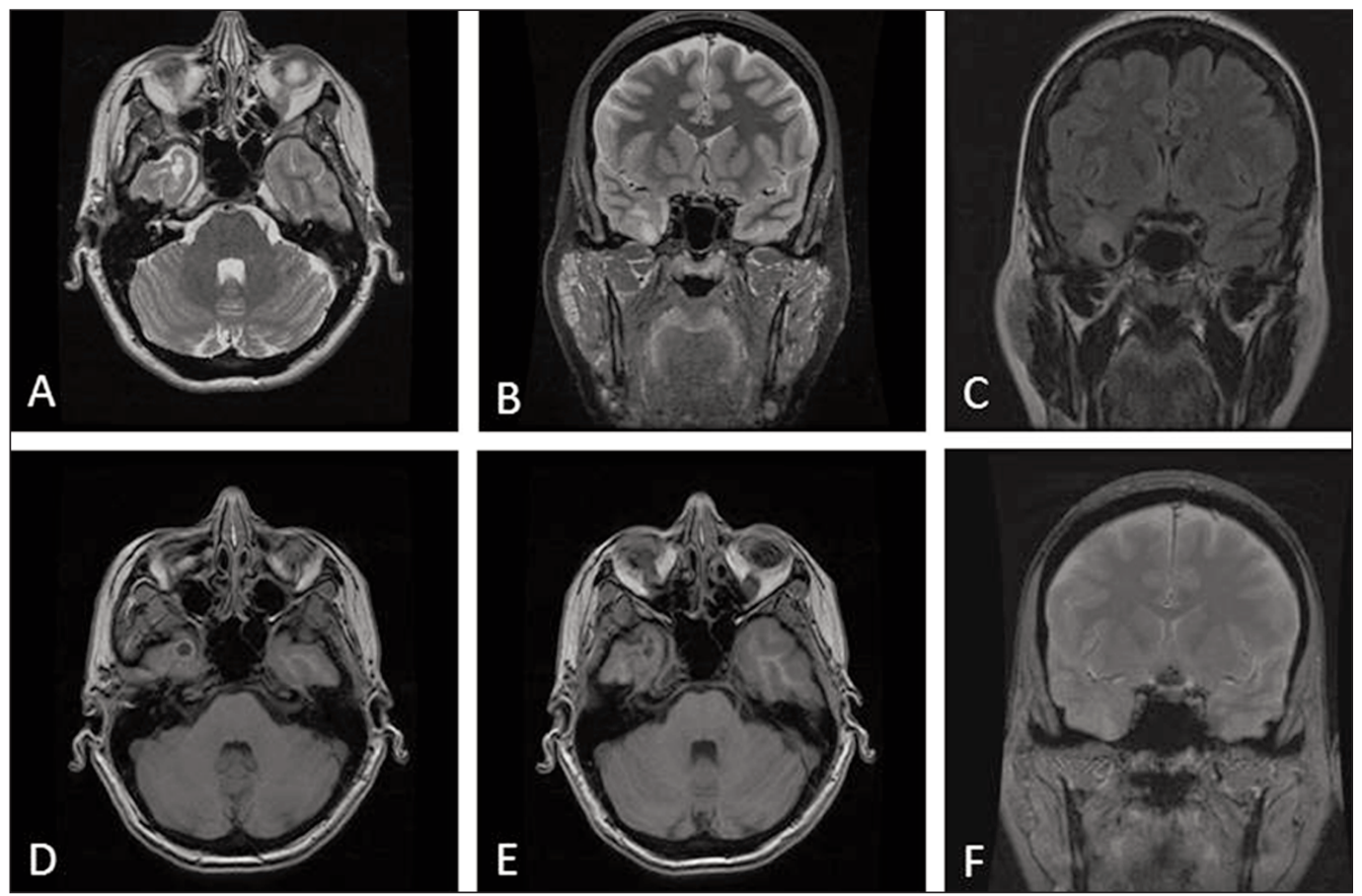

Figure 2: MRI of the brain: A) Axial Fast Spin Echo (FSE), B) Coronal FSE, C) Coronal Fluid Attenuation Inversion Recovery (FLAIR), D) and E) Axial T1 without gadolinium, F) Coronal gradient echo. These images demonstrate a predominantly cystic lesion in the right temporal pole. It has mild local mass effect and measures approximately $2.5 \mathrm{~cm}$. in maximum diameter. There are 2 cystic components, with mild surrounding cortical thickening and $T 2$ hyperintensity. There is mild T1 hyperintensity surrounding the cysts, no enhancement with gadolinium and no magnetic susceptibility.

treatment ${ }^{4}$. My final impression is medically-intractable right temporal lobe epilepsy due to a low-grade primary tumour, most likely a ganglioglioma.

\section{Dr. STEVEN}

The patient underwent a right temporal lobectomy including amygdalohippocampectomy. Although the external surface of the brain was normal, the pia mater in the region of the lesion was abnormally thin and friable, something which is often seen adjacent to chronic lesions. The lesion was cystic with surrounding abnormal appearing brain. No abnormalities were noted in the hippocampus or amygdala.

\section{Dr. AlSufiani}

The specimen was serially sampled. Routine stains identified a moderately cellular fibrillary lesion largely within temporal white matter (Figure 4). It was relatively well demarcated from adjacent parenchyma. Lesional cells displayed fascicular and radial angiocentric growth patterns with representative cells having elongated oval nuclei and coarse bipolar processes bearing abundant glial fibrillary acidic protein (GFAP) (Figure 4). Angiocentric growth patterns dominated peripherally while fascicular patterns dominated centrally. Tiny lacunes were noted within the lesion and some of these were finely decorated by epithelial membrane antigen (EMA) expression (Figure 4). Ultrastructural examination revealed small cilia-lined spaces, associated basal bodies and lesional cells bearing abundant intermediate filaments (Figure 4). Mesial temporal structures were relatively well preserved, with only mild gliosis.

The final diagnosis was angiocentric glioma with mild reactive changes in mesial temporal structures (consistent with chronic seizure activity).

\section{Dr. MCLaChlan}

At her last follow-up 1.5 years after surgery she was free of seizures on a lower dose of levetiracetam $250 \mathrm{mg}$ twice daily. She had a transient decrease in short term memory and mild postoperative depression both of which resolved within a year of surgery. She is driving and working full time.

\section{Dr. AlSufiani}

Angiocentric gliomas ${ }^{5-8}$ are rare low-grade World Health Organization (WHO) grade I tumours with an excellent

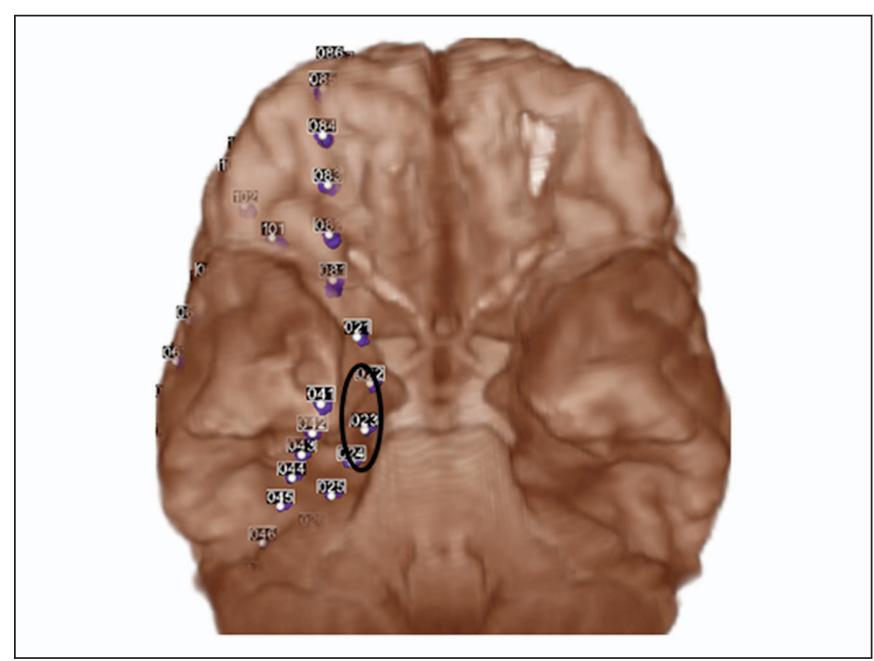

Figure 3: Subdural electrode map showing lead placements and seizure origin (black oval). 


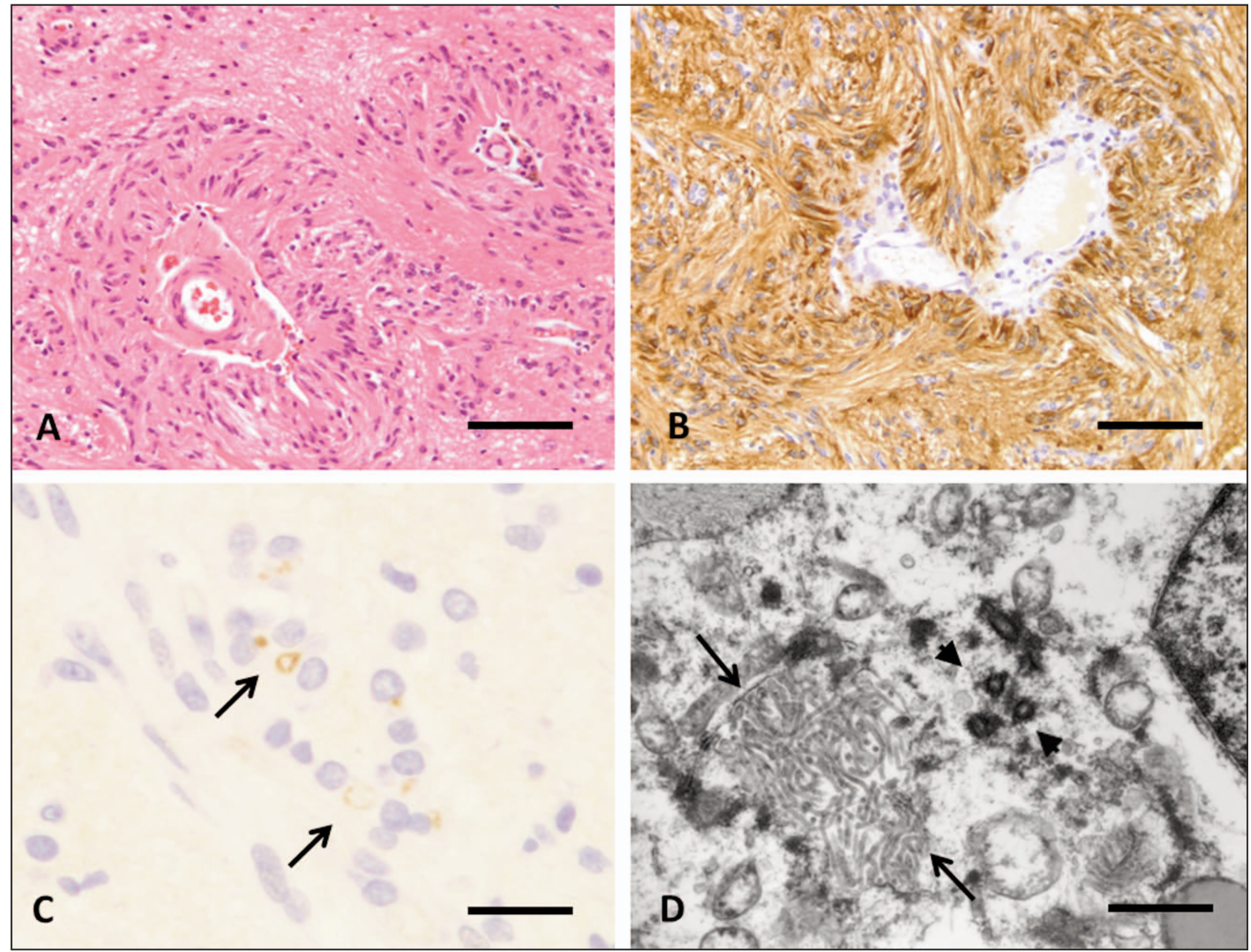

Figure 4: A) Radial angiocentric patterning of tumour cells $(H \& E$, bar $=100$ um), B) Tumour cell expression of glial fibrillary acidic protein (anti-GFAP, immunoperoxidase, bar=100um), C) Focal tumour cell expression of epithelial membrane antigen in microlumens (anti-EMA, immunoperoxidase, bar=50 um), D) Microvilli (arrow) and basal bodies (arrowheads) as demonstrated by transmission electron microscopy (uranyl acetate /lead citrate preparation, bar $=1 \mathrm{um}$ ) prognosis that are very recent additions to the WHO classification of tumours of the central nervous system. They typically arise in the setting of chronic epilepsy. While the average age at diagnosis is 17 , the reported age range extends from early childhood to the eighth decade ${ }^{8}$. Angiocentric gliomas tend to be superficial in location and have relatively characteristic MRI signatures: well-demarcated, hyperintense solid lesions by FLAIR sequences.

The tumours display a striking radial angiocentric architecture with perivascular pseudorosettes. Individual lesional cells are monotonous and bipolar with slender, granular nuclei. Expression of GFAP, vimentin and S100 protein are typical. EMA and CD99 expression is focal and more variable. There is no expression of neuronal markers among lesional cells. Ultrastructural examinations have revealed microlumens hosting cilia and/or microvilli with adjacent cells bound by junctional complexes. Together, these features have suggested an ependymal lineage to some, but their usual cortical location has led others to suggest radial glia as a more likely source. A consistent cytogenetic or molecular genetic profile has not been described for angiocentric gliomas and undoubtedly such studies are hindered by a paucity of cases.

\section{Dr. Burneo}

The final pathology identified an unusual type of a low-grade glioma, nonetheless with excellent prognosis.

In regards to discontinuation of AEDs after surgery, it is my practice to keep the patient on AEDs, at the lowest possible dose of monotherapy. Evidence on how to proceed with AEDs after surgery in the long term is lacking, and invites a much needed randomized clinical trial.

\section{REFERENCES}

1. Blume WT, Luders HO, Mizrahi E, Tassinari C, van Emde Boas W, Engel J, Jr. Glossary of descriptive terminology for ictal semiology: Report of the ILAE task force on classification and terminology. Epilepsia. 2001;42(9):1212-8.

2. Barkovich AJ, Kuzniecky RI, Jackson GD, Guerrini R, Dobyns WB. A developmental and genetic classification for malformations of cortical development. Neurology. 2005;65(12): 1873-87.

3. Kwan P, Arzimanoglou A, Berg AT, et al. Definition of drug resistant epilepsy: Consensus proposal by the ad hoc task force of the ILAE commission on therapeutic strategies. Epilepsia. 2010;51(6):1069-77.

4. Wiebe S, Blume WT, Girvin JP, Eliasziw M, Effectiveness and Efficiency of Surgery for Temporal Lobe Epilepsy Study Group. A randomized, controlled trial of surgery for temporal-lobe epilepsy. N Engl J Med. 2001;345(5):311-8.

5. Preusser M, Hoischen A, Novak K, et al. Angiocentric glioma: Report of clinico-pathologic and genetic findings in 8 cases. Am J Surg Pathol. 2007;31(11):1709-18.

6. Marburger T, Prayson R. Angiocentric glioma: A clinicopathologic review of 5 tumors with identification of associated cortical dysplasia. Arch Pathol Lab Med. 2011;135(8):1037-41.

7. Louis DN, Ohgaki H, Wiestler OD, et al. The 2007 WHO classification of tumours of the central nervous system. Acta Neuropathol. 2007;114(2):97-109.

8. Brat DJ, Scheithauer BW, Fuller GN, Tihan T. Newly codified glial neoplasms of the 2007 WHO classification of tumours of the central nervous system: Angiocentric glioma, pilomyxoid astrocytoma and pituicytoma. Brain Pathol. 2007;17(3):319-24. 